\title{
Fixed Point Theory in Modular Function Spaces
}

\author{
Mohamed Amine Khamsi² \\ Department of Mathematical Sciences \\ The University of Texas at El Paso \\ El Paso, Texas 79968, USA.
}

The author would like to thank Colegio El Pinar for their hospitality during the preparation of this survey.

\section{Introduction}

First attemps to generalize the classical function spaces of the Lebesgue type $L^{p}$ were made in the early 1930's by Orlicz and Birnbaum ${ }^{1}$ in connection with orthogonal expansions. Their approach consisted in considering spaces of functions with some growth properties different from the power type growth control provided by the $L^{p}$-norms. Namely, they considered the function spaces defined as follows:

$$
L^{\varphi}=\left\{f: \Re \rightarrow \Re ; \exists \lambda>0: \int_{\Re} \varphi(\lambda|f(x)|) d x<\infty\right\},
$$

where $\varphi:[0, \infty] \rightarrow[0, \infty]$ was assumed to be a convex function increasing to infinity, i.e. the function which to some extent behaves similarly to power functions $\varphi(t)=t^{p}$. Later on, the assumption of convexity for Orlicz functions $\varphi$ was frequently omitted. Let us mention two typical examples of such

\footnotetext{
${ }^{1}$ The author would like to apologize to the reader for not including the references whithin the paper.
} 
functions:

$$
\varphi(t)=e^{t}-1, \quad \varphi(t)=\ln (1+t) .
$$

The possibility of introducing the structure of a linear metric in $L^{\varphi}$ as well as the interesting properties of these spaces and many applications to differential and integral equations with kernels of nonpower types were among the reasons for the development of the theory of Orlicz spaces, their applications and generalizations for more than half of the century. Recent interest in classical Orlicz spaces has emerged in connection with problems of convexity, the Boyd indices and rearrangement invariant function spaces.

We may observe two principal directions of further development. The first one is a theory of Banach function spaces initiated in 1955 by Luxemburg and then developped in a series of joint papers with Zaanen. The main idea of that theory consists in considering a function space $L$ of all functions $f: X \rightarrow \Re, f \in M(X, \Re)$, such that $\|f\|<\infty$, where $(X, \Sigma, \mu)$ is a measure space, $M(X, S)$ denotes the space of all strongly measurable functions acting from $X$ into a Banach space $S$ and $\|$.$\| is a function norm which satisfies$

$$
\|f\| \leq\|g\| \text { whenever }|f(x)| \leq|g(x)| \mu \text {-a.e. }
$$

The other way, also inspired by the successful theory of Orlicz spaces, is based on replacing the particular, integral form of the nonlinear functional, which controls the growth of members of the space, by an abstractly given functional with some good properties. This idea was the basis of the theory of modular spaces initiated by Nakano in 1950 in connection with the theory of order spaces and redefined and generalized by Luxemburg and Orlicz in 1959. Let us give a brief account of some basic facts of their theory.

Let $\mathcal{X}$ be a vector space over $\Re$ (we may take the complex field as the scalar set for the vector space). A functional $\rho: \mathcal{X} \rightarrow[0, \infty]$ is called a pseudomodular, if for arbitrary $f$ and $g$, elements of $\mathcal{X}$, there holds :

(1) $\rho(0)=0$;

(2) $\rho(\alpha f)=\rho(f)$ whenever $|\alpha|=1$,

(3) $\rho(\alpha f+\beta g) \leq \rho(f)+\rho(g)$ whenever $\alpha, \beta \geq 0$ and $\alpha+\beta=1$. 
If we replace (3) by

(3') $\rho(\alpha f+\beta g) \leq \alpha \rho(f)+\beta \rho(g)$ whenever $\alpha, \beta \geq 0$ and $\alpha+\beta=1$.

then the pseudomodular $\rho$ is called convex. If in place of (1) there holds

(1') $\rho(0)=0$; and $\rho(\lambda f)=0$ for all $\lambda>0$ implies $f=0$,

then $\rho$ is called a semimodular. If moreover,

(1") $\rho(f)=0$ if and only if $f=0$

then $\rho$ is called a modular. If $\rho$ is a pseudomodular in $\mathcal{X}$ then the set defined by

$$
\mathcal{X}_{\rho}=\left\{h \in \mathcal{X} ; \lim _{\lambda \rightarrow 0} \rho(\lambda h)=0\right\}
$$

is called a modular space. $\mathcal{X}_{\rho}$ is a vector subspace of $\mathcal{X}$. For a pseudomodular $\rho$ in $\mathcal{X}$ we may define an $\mathrm{F}$-seminorm by the formula :

$$
\|f\|_{\rho}=\inf \left\{t>0 ; \rho\left(\frac{f}{t}\right) \leq t\right\} .
$$

If $\rho$ is a convex pseudomodular then the functional given by

$$
\|f\|_{\rho}=\inf \left\{t>0 ; \rho\left(\frac{f}{t}\right) \leq 1\right\} .
$$

is a seminorm. Observe that the previous formulas define F-norms and norms respectively, if $\rho$ is a modular. One can check that

$$
\left\|f_{n}-f\right\|_{\rho} \rightarrow 0 \text { is equivalent to } \rho\left(t\left(f_{n}-f\right)\right) \rightarrow 0 \text { for all } t>0 .
$$

It is also an important fact that $\rho(f) \leq\|f\|_{\rho}$ provided $\|f\|_{\rho}<1$.

We say that a sequence $\left(f_{n}\right)$ is modular convergent (briefly: $\rho$-convergent) to $f \in \mathcal{X}_{\rho}$ if there exists a $\lambda>0$ such that $\rho\left(\lambda\left(f_{n}-f\right)\right) \rightarrow 0$ as $n \rightarrow \infty$. A modular $\rho$ is called :

(a) Right-continuous, if for all $f \in \mathcal{X}_{\rho}$, we have

$$
\lim _{t \rightarrow 1+} \rho(t f)=\rho(f)
$$


(b) Left-continuous, if for all $f \in \mathcal{X}_{\rho}$, we have

$$
\lim _{t \rightarrow 1-} \rho(t f)=\rho(f)
$$

(c) Continuous, if it is both right and left continuous.

In this way, the Orlicz space becomes a modular space, where $\mathcal{X}=$ $M(X, \Re)$ and the modular $\rho$ is defined by

$$
\rho(f)=\int_{\Re} \varphi(|f(x)|) d x .
$$

On the base of the modular theory Musielak and Orlicz founded in 1959 a theory of Musielak-Orlicz spaces, i.e. the modular spaces induced by modulars of the following form:

$$
\rho(f)=\int_{\Re} \varphi(x,|f(x)|) d x .
$$

where $\varphi: X \times \Re^{+} \rightarrow \Re^{+}$is a function, continuous and increasing to infinity in the second variable, and is measurable in the first one. Such spaces have been studied for almost forty years and there is known a large set of applications of such spaces in variuous parts of analysis. They were also generalized in many directions, e.g. some generalizations to the case of vector valued functions have been considered and many authors have investigated spaces generated by families of Musielak-Orlicz modulars. Such spaces have many applications in probability and mathematical statistics.

We may observe, however, the situation where on the one hand we have a very abstract general theory of modular spaces which cannot give proper answers to many interesting questions and, on the other hand, spaces constructed on the image of Musielak-Orlicz spaces. In the latter case, the concepts from Musielak-Orlicz theory do not fit the new demands. Another common difficulty consists in the fact that the theory of Musielak-Orlicz spaces, though very useful, is not structural, in the sense that many operations like taking sums or passing to equivalent modulars lead beyond the class of Musielak-Orlicz spaces.

In this work, we will investigate modular spaces which lies somewhere in between, i.e. class of modular spaces given by modulars or semimodulars not 
of any particular forms but, nevertheless, having much more convenient properties than the abstract modulars can possess. In other words, we present a useful tool for applications whenever there is a need to introduce a function space by means of functionals which have some reasonable properties but which are far from being norms or F-norms.

\section{Bibliographical remarks}

The monographic exposition of the theory of Orlicz spaces may be found in the book of Krasnosel'skii and Rutickii. For a current review of the theory of Musielak-Orlicz spaces and modular spaces the reader is referred to the book of Musielak and the most recent book of Kozlowski.

\section{Basic definitions and results}

We start with a brief recollection of basic concepts and facts of the theory of modular spaces as formulated by Kozlowski.

Definition 1. Let $X$ be an arbitrary vector space.

(a) A functional $\rho: X \rightarrow[0, \infty]$ is called a modular if for arbitrary x,y in $\mathrm{X}$,

(i) $\rho(x)=0$ iff $x=0$,

(ii) $\rho(\alpha x)=\rho(x)$ for every scalar $\alpha$ with $|\alpha|=1$,

(iii) $\rho(\alpha x+\beta y) \leq \rho(x)+\rho(y)$ if $\alpha+\beta=1$ and $\alpha \geq 0, \beta \geq 0$.

(b) If (iii) is replaced by

(iii)' $\rho(\alpha x+\beta y) \leq \alpha \rho(x)+\beta \rho(y)$ if $\alpha+\beta=1$ and $\alpha \geq 0, \beta \geq 0$,

we say that $\rho$ is a convex modular.

(c) A modular $\rho$ defines a corresponding modular space, i.e the vector space $X_{\rho}$ given by

$$
X_{\rho}=\{x \in X ; \rho(\lambda x) \rightarrow 0 \text { as } \lambda \rightarrow 0\}
$$


In general the modular $\rho$ is not subadditive and therefore does not behave as a norm or a distance. But one can associate to a modular an $F$-norm. Recall that a functional $\|\|:. X \rightarrow[0, \infty]$ defines an $F$-norm if and only if

(1) $\|x\|=0$ if and only if $x=0$,

(2) $\|\alpha x\|=\|x\|$ whenever $|\alpha|=1$,

(3) $\|x+y\| \leq\|x\|+\|y\|$,

(4) $\left\|\alpha_{n} x_{n}-\alpha x\right\| \rightarrow 0$ if $\alpha_{n} \rightarrow \alpha$ and $\left\|x_{n}-x\right\| \rightarrow 0$.

An $F$-norm defines a distance on $X$ by

$$
d(x, y)=\|x-y\| .
$$

The linear metric space $(X, d)$ is called an $F$-space if $d$ is complete.

Definition 2. The modular space $X_{\rho}$ can be equipped with an $F$-norm defined by

$$
\|x\|_{\rho}=\inf \left\{\alpha>0 ; \rho\left(\frac{x}{\alpha}\right) \leq \alpha\right\} .
$$

When $\rho$ is convex the formula

$$
\|x\|_{\rho}=\inf \left\{\alpha>0 ; \rho\left(\frac{x}{\alpha}\right) \leq 1\right\} .
$$

defines a norm which is frequently called the Luxemburg norm.

It is clear that $\left\|x_{n}\right\|_{\rho} \rightarrow 0$ if and only if $\rho\left(\beta x_{n}\right) \rightarrow 0$ for every $\beta>0$. One can easily observe that $\alpha \rightarrow \rho(\alpha x)$ is increasing for every $x \in X$.

As a classical example we may give the Orlicz' modular defined for every measurable real function $f$ by the formula

$$
\rho(f)=\int_{\Re} \varphi(|f(t)|) d m(t),
$$

where $m$ denotes the Lebesgue measure in $\Re$ and $\varphi: \Re \rightarrow[0, \infty)$ is continuous, $\varphi(0)=0$ and $\varphi(t) \rightarrow \infty$ as $t \rightarrow \infty$.

The modular space induced by the Orlicz' modular $\rho_{\varphi}$ is called the Orlicz space $L^{\varphi}$. 
Let $\Omega$ be a nonempty set and $\Sigma$ be a nontrivial $\sigma$-algebra of subsets of $\Omega$. Let $\mathcal{P}$ be a $\delta$-ring of subsets of $\Sigma$, such that $E \cap A \in \mathcal{P}$ for any $E \in \mathcal{P}$ and $A \in \Sigma$. Let us assume that there exists an increasing sequence of sets $K_{n} \in \mathcal{P}$ such that $\Omega=\bigcup K_{n}$. In an other word, the family $\mathcal{P}$ plays the role of the $\delta$-ring of subsets of finite measure. By $\mathcal{E}$ we denote the linear space of all simple functions with supports from $\mathcal{P}$. By $\mathcal{M}$ we will denote the space of all measurable functions, i.e. all functions $f: \Omega \rightarrow \Re$ such that there exists a sequence $\left\{g_{n}\right\} \in \mathcal{E},\left|g_{n}\right| \leq|f|$ and $g_{n}(\omega) \rightarrow f(\omega)$ for all $\omega \in \Omega$. By $1_{A}$ we denote the characteristic function of the set $A$.

Let us add that a set function $\mu: \Sigma \rightarrow[0, \infty]$ is called a $\sigma$-subadditive measure if

(i) $\mu(\emptyset)=0$,

(ii) $\mu(A) \leq \mu(B)$ for any $A \subset B$,

(iii) $\mu\left(\bigcup A_{n}\right) \leq \sum \mu\left(A_{n}\right)$ for any sequence of sets $A_{n} \in \Sigma$.

Definition 3. A functional $\rho: \mathcal{E} \times \Sigma \rightarrow[0, \infty]$ is called a function modular if

$\left(P_{1}\right) \rho(0, E)=0$ for any $E \in \Sigma$,

$\left(P_{2}\right) \rho(f, E) \leq \rho(g, E)$ whenever $|f(\omega) \leq| g(\omega) \mid$ for any $\omega \in \Omega, f, g \in \mathcal{E}$ and $E \in \Sigma$,

$\left(P_{3}\right) \rho(f,):. \Sigma \rightarrow[0, \infty]$ is a $\sigma$-subadditive measure for every $f \in \mathcal{E}$,

$\left(P_{4}\right) \rho(\alpha, A) \rightarrow 0$ as $\alpha$ decreases to 0 for every $A \in \mathcal{P}$, where $\rho(\alpha, A)=$ $\rho\left(\alpha 1_{A}, A\right)$,

$\left(P_{5}\right)$ if there exists $\alpha>0$ such that $\rho(\alpha, A)=0$, then $\rho(\beta, A)=0$ for every $\beta>0$,

$\left(P_{6}\right)$ for any $\alpha>0 \rho(\alpha,$.$) is order continuous on \mathcal{P}$, that is $\rho\left(\alpha, A_{n}\right) \rightarrow 0$ if $\left\{A_{n}\right\} \in \mathcal{P}$ and decreases to $\emptyset$.

The definition of $\rho$ is then extended to $f \in \mathcal{M}$ by

$$
\rho(f, E)=\sup \{\rho(g, E) ; g \in \mathcal{E},|g(\omega)| \leq|f(\omega)| \omega \in \Omega\} .
$$


This will enable us to define $\rho(\alpha, E)$ for sets $E$ not in $\mathcal{P}$; for the sake of simplicity, we write $\rho(f)$ instead of $\rho(f, \Omega)$.

Definition 4. A set $E$ is said to be $\rho$-null if and only if $\rho(\alpha, E)=0$ for $\alpha>0$. A property $p(\omega)$ is said to hold $\rho$-almost everywhere $(\rho$-a.e.) if the set $\{\omega \in \Omega ; p(\omega)$ does not hold $\}$ is $\rho$-null. For example we will say frequently $f_{n} \rightarrow f \rho$-a.e.

Note that a countable union of $\rho$-null sets is still $\rho$-null. In the sequel we will identify sets $A$ and $B$ whose symmetric difference $A \Delta B$ is $\rho$-null; similarly we will identify measurable functions which differ only on a $\rho$-null set.

It is easy to see that the functional $\rho: \mathcal{M} \rightarrow[0, \infty]$ is a modular in the sense of Definition 1. The modular space determined by $\rho$ will be called a modular function space and will be denoted by $L_{\rho}$. Recall that

$$
L_{\rho}=\left\{f \in \mathcal{M} ; \lim _{\alpha \rightarrow 0} \rho(\alpha f)=0\right\} .
$$

Let us recall some basic facts about modular function spaces.

\section{Theorem 1.}

(1) $\left(L_{\rho},\|.\|_{\rho}\right)$ is a complete space and the F-norm $\|.\|_{\rho}$ is monotone with respect to the natural order in $\mathcal{M}$.

(2) If there is a number $\alpha>0$ such that $\rho\left(\alpha\left(f_{n}-f\right)\right) \rightarrow 0$ then there exists a subsequence $\left\{g_{n}\right\}$ of $\left\{f_{n}\right\}$ such that $g_{n} \rightarrow f \rho$-a.e.

(3) (Egoroff's Theorem) If $f_{n} \rightarrow f \rho$-a.e. then there exists an increasing sequence of sets $H_{k} \in \mathcal{P}$ such that $\Omega=\bigcup H_{k}$ and $\left\{f_{n}\right\}$ converges uniformly to $f$ on every $H_{k}$.

(4) Define

$$
L_{\rho}^{0}=\{f \in \mathcal{M} ; \rho(f, .) \text { is order continuous }\}
$$

and

$$
E_{\rho}=\left\{f \in \mathcal{M} ; \alpha f \in L_{\rho}^{0} \text { for every } \alpha>0\right\} .
$$

Then 
(4.1) $E_{\rho} \subset L_{\rho}^{0} \subset L_{\rho}$,

(4.2) $E_{\rho}$ has the Lebesgue property, i.e. $\left\|f 1_{D_{n}}\right\|_{\rho} \rightarrow 0$ if $f \in E_{\rho}$ and $D_{n}$ decreases to $\emptyset$,

(4.3) $E_{\rho}$ is the closure of $\mathcal{E}$ (in the sense of $\|.\|_{\rho}$ ).

(5) (Vitali's Theorem) If $f_{n} \in E_{\rho}$ and $f_{n} \rightarrow f \in L_{\rho} \rho$-a.e., then the following conditions are equivalent

(i) $f \in E_{\rho}$ and $\left\|f_{n}-f\right\|_{\rho} \rightarrow 0$,

(ii) for every $\alpha>0$ the subadditive measures $\rho\left(\alpha f_{n},.\right)$ are equicontinuous, i.e.

$$
\lim _{k \rightarrow \infty} \sup _{n} \rho\left(\alpha f_{n}, D_{k}\right)=0,
$$

for every sequence $\left\{D_{k}\right\} \in \Sigma$ that decreases to $\emptyset$.

(6) (Lebesgue's Theorem) If $f_{n}, f \in \mathcal{M}, f_{n} \rightarrow f \rho$-a.e. and there exists a function $g \in E_{\rho}$ such that $\left|f_{n}\right| \leq|g| \rho$-a.e. for all $n$, then $\left\|f_{n}-f\right\|_{\rho} \rightarrow 0$.

(7) For $f_{n}, f \in \mathcal{M}$, the following conditions are equivalent

(i) $\rho$ has the Fatou property, i.e.

$$
\rho\left(f_{n}\right) \uparrow \rho(f) \text { whenever }\left|f_{n}\right| \uparrow|f| \rho-\text { a.e. }
$$

(ii) $\rho$ is a left continuous modular, i.e.

$$
\rho\left(\alpha_{n} f\right) \uparrow \rho(f) \text { whenever } \alpha_{n} \uparrow 1 .
$$

(iii) $\rho(f) \leq \liminf \rho\left(f_{n}\right)$ whenever $f_{n} \rightarrow f \rho$-a.e.

(8) A function modular is said to satisfy the $\Delta_{2}$-condition if $\sup \rho\left(2 f_{n}, D_{k}\right) \rightarrow$ 0 as $k \rightarrow \infty$ whenever $D_{k}$ decreases to $\emptyset$ and $\sup \rho\left(f_{n}, D_{k}\right) \rightarrow 0$.

It is known that $\Delta_{2}$ is equivalent to the equality $E_{\rho}=L_{\rho}$. The other characterization is as follows: $\rho$ satisfies the $\Delta_{2}$ condition if and only if $F$ norm convergence and modular convergence are equivalent.

\section{Definition 5.}

(a) The sequence $\left\{f_{n}\right\} \subset L_{\rho}$ is said to be $\rho$-convergent to $f \in L_{\rho}$ if $\rho\left(f_{n}-\right.$ $f) \rightarrow 0$ as $n \rightarrow \infty$, 
(b) The sequence $\left\{f_{n}\right\} \subset L_{\rho}$ is said to be $\rho$-Cauchy if $\rho\left(f_{n}-f_{m}\right) \rightarrow 0$ as $n$ and $m$ go to $\infty$,

(c) A subset $C$ of $L_{\rho}$ is called $\rho$-closed if the $\rho$-limit of a $\rho$-convergent sequence of $C$ always belongs to $\mathrm{C}$.

(d) A subset $C$ of $L_{\rho}$ is called $\rho$-compact if every sequence in $C$ has a $\rho$-convergent subsequence in $C$.

(e) A subset $C$ of $L_{\rho}$ is called $\rho$-bounded if

$$
\delta_{\rho}(C)=\sup \{\rho(f-g) ; f, g \in C\}<\infty,
$$

(f) Let $f \in L_{\rho}$ and $C \subset L_{\rho}$. Define the $\rho$-distance between $f$ and $C$ as :

$$
\delta_{\rho}(f, C)=\inf \{\rho(f-g) ; g \in C\} .
$$

The above terminology is used because of its formal similarity to the metric case. Since $\rho$ does not behave in general as a distance, one should be very careful when dealing with these notions. In particular, $\rho$-convergence does not imply $\rho$-Cauchy since $\rho$ does not satisfy the triangle inequality. In fact, one can show that this will happen if and only if $\rho$ satisfies the $\Delta_{2}$-condition.

\section{Remarks.}

(1) Since the intersection of $\rho$-closed sets is $\rho$-closed, one can associate to any subset $A \subset L_{\rho}$, a $\rho$-closed subset, denoted $\bar{A}$, which is minimal in the following sense:

$$
\text { if } A \subset B \text { and } B \text { is } \rho \text {-closed, then } \bar{A} \subset B
$$

We will call $\bar{A}$ the $\rho$-closure of $A$.

(2) We have an easy characterization of the Fatou property in terms of $\rho$ balls. Indeed, one can easily check that $\rho$-balls are $\rho$-closed if and only if $\rho$ has the Fatou property. Recall the definition of the $\rho$-ball $B_{\rho}(f, r)$, where $f \in L_{\rho}$ and $r \geq 0$, as

$$
B_{\rho}(f, r)=\left\{h \in L_{\rho} ; \rho(f-h) \leq r\right\} .
$$

$f$ and $r$ are called respectively the center and the radius of the $\rho$-ball $B_{\rho}(f, r)$. 
In Banach spaces, when we think of reflexivity automatically the dual space is present in our taught. But in modular spaces, it is very hard to conceive the dual space. To circumvent the problem, we use some characterizations of reflexivity.

Definition 6. Let $X_{\rho}$ be a modular space.

(a) We will say that $X_{\rho}$ or $\rho$ satisfy the property $(R)$ if and only if every decreasing sequence of nonempty $\rho$-closed and $\rho$-bounded convex subset of $X_{\rho}$, has a nonempty intersection.

(b) We will say that $X_{\rho}$ or $\rho$ satisfy the property $\left(R^{\prime}\right)$ if and only if for every $\rho$-bounded sequence $\left(f_{n}\right) \subset X_{\rho}$, there exists a subsequence $\left(f_{n^{\prime}}\right)$ of $\left(f_{n}\right)$ such that the intersection of $\left(\operatorname{cl}\left(\operatorname{conv}\left\{f_{i^{\prime}} ; i \geq n\right\}\right)\right)$ is nonempty and reduced to one point.

By $\operatorname{cl}(\operatorname{conv}\{A\})$, we mean the $\rho$-closure of the smallest convex subset containing $A$. Clearly the property $\left(R^{\prime}\right)$ implies the property $(R)$ and are equivalent in Banach spaces.

Before we give few examples of modular function spaces we will need the following definition.

Definition 7. Let $(\Omega, \Sigma, \mu)$ be a measure space. A real function $\varphi$ defined on $\Omega \times \Re_{+}$will be said to belong to the class $\Phi$ if the following conditions are satisfied :

(i) $\varphi(\omega, u)$ is a nondecreasing continuous function of $u$ such that $\varphi(\omega, 0)=$ $0, \varphi(\omega, u)>0$ for $u>0$ and $\varphi(\omega, u) \rightarrow \infty$ as $u \rightarrow \infty$,

(ii) $\varphi(\omega, u)$ is a $\Sigma$-measurable function of $\omega$ for all $u \geq 0$,

(iii) $\varphi(\omega, u)$ is a convex function of $u$, for all $\omega \in \Omega$.

For the sake of generality some authors will not assume that $\varphi(\omega, u)$ is a convex function of $u$. Although the results in this work can be easily generalized into their setting, it is not the feeling of the author that this will change anything to the general idea.

\section{Examples.}


(1) It is easy to check that Orlicz spaces are modular function spaces. Similarly Musielak-Orlicz spaces, i.e. spaces determined by a modular of the form

$$
\rho(f, E)=\int_{E} \varphi(t,|f(t)|) d \mu(t),
$$

are modular function spaces, provided $\varphi$ belongs to the class $\Phi$. For the precise definitions and properties of Musielak-Orlicz spaces see the book by Musielak, where they are called generalized Orlicz spaces. The particular case when

$$
\varphi(t, s)=s^{p}, \quad \text { for } 1 \leq p<\infty,
$$

gives the classical $L^{p}$-spaces. The Luxemburg's norm is the classical $L^{p}$-norm. Moreover we have

$$
\rho(f)=\|f\|_{L^{p}}^{p}
$$

Let us add that Musielak-Orlicz modular spaces are complete for the modular.

(2) Suppose $\mathcal{M}$ is a family of $\sigma$-additive measures on $(\Omega, \Sigma)$, and $\varphi \in \Phi$. One can prove that

$$
\rho(f, E)=\sup _{\tau \in \mathcal{M}} \int_{E} \varphi(t,|f(t)|) d \mu(t)
$$

is a function modular. As an example of function spaces determined by a function modular of this type we can mention the Lorentz type $L^{p}$-spaces, where

$$
\rho(f, E)=\sup _{\tau \in \mathcal{T}} \int_{E}|f(t)|^{p} d \mu_{\tau}(t) .
$$

Here $\mu$ is a fixed $\sigma$-finite measure on $\Omega, \mathcal{T}$ is any set of measurable, invertible transformations $\tau: \Omega \rightarrow \Omega$ and $\mu_{\tau}(E)=\mu\left(\tau^{-1}(E)\right)$.

\section{Some fixed points theorems in Modular spaces}

In this section we discuss the existence of fixed points for mappings which are nonexpansive or contractive in the modular sense. Certainly, one can 
also consider mappings which are contractive with respect to the $F$-norm induced by the modular. It is worth to mention that, generally speaking, there is no natural relation between the two kinds of nonexpansiveness. Once again we would like to emphasize our philosophy that all the results expressed in terms of modulars are more convenient in the sense that their assumptions are much easier to verify.

Definition 8. Let $C$ be a subset of a modular space $L_{\rho}$ and let $T: C \rightarrow C$ be an arbitrary mapping.

(1) $T$ is a $\rho$-contraction if there exists $\lambda<1$ such that

$$
\rho(T(f)-T(g)) \leq \lambda \rho(f-g)
$$

for all $f, g \in C$.

(2) $T$ is said to be $\rho$-nonexpansive if

$$
\rho(T(f)-T(g)) \leq \rho(f-g)
$$

for all $f, g \in C$.

(3) $f \in C$ is said to be a fixed point of $T$ if $T(f)=f$. The fixed point set of $T$ will be denoted Fix $(T)$.

$C$ will be said to have the fixed point property if every $\rho$-nonexpansive selfmap defined on $C$ has a fixed point.

An analog to banach contraction principle, can be stated as follows.

Theorem 2. Let $C$ be $\rho$-complete $\rho$-bounded subset of $L_{\rho}$ and $T: C \rightarrow C$ be a $\rho$-strict contraction. Then $T$ has a unique fixed point $z \in C$. Moreover $z$ is the $\rho$-limit of the iterate of any point in $C$ under the action of $T$.

Recall that a subset $D$ of $L_{\rho}$ is said to be $\rho$-complete if every $\rho$-Cauchy sequence from $D$ is convergent in $D$.

We may relax the assumption regarding the boundedness of $C$ and assume there exists a bounded orbit instead. In this case, the uniqueness of the fixed point is dropped and replaced by

if $f$ and $g$ are two fixed points of $T$ such that $\rho(f-g)<\infty$, then $f=g$. 
In order to discuss our first result regarding $\rho$-nonexpansive mappings, we need the following definition.

Definition 9. The growth function $w_{\rho}$ of a function modular $\rho$ is defined as follows:

$$
w_{\rho}(t)=\sup \left\{\frac{\rho(t f)}{\rho(f)} ; f \in L_{\rho}, 0<\rho(f)<\infty\right\}, t \geq 0 .
$$

Observe that $w_{\rho}(t) \leq 1$ for all $t \in[0,1]$.

Definition 10. We say that $\rho$ satisfies the regular growth condition if $w_{\rho}(t)<$ 1 for all $t \in[0,1)$.

The class of function modulars that satisfies the regular growth condition is quite large. For instance, if $\rho$ is convex that $\rho(t f) \leq t \rho(f)$ for $t \in[0,1]$, and consequently $w_{\rho}(t) \leq t<1$ for $t<1$. Thus all convex function modulars satisfy the regular growth condition. It is not hard to prove that if $\rho$ is an Orlicz modular then, in the case of finite measure, $\rho$ satisfies the regular growth condition if and only if

$$
\limsup _{s \rightarrow \infty} \frac{\varphi(t s)}{\varphi(s)}<1, \quad \text { for all } t \in[0,1)
$$

where $\varphi$ denotes the Orlicz function associated with $\rho$. If there exists a constant $K>0$ such that $\rho(2 f) \leq K \rho(f)$ for all $f \in L_{\rho}$, then $w_{\rho}$ is submultiplicative and hence there exists $p>1$ such that $w_{\rho}(t) \leq t^{p}$ for $t<1$. Consequently, such function modulars also satisfy the regular growth condition.

Recall that a set $B \subset L_{\rho}$ is said to be star-shaped if there exists $f \in B$ such that $f+\lambda(g-f) \in B$ whenever $\lambda \in[0,1]$ and $g \in B$.

Theorem 3. Assume that $\rho$ has Fatou property and satisfies the regular growth condition. Let $B$ be a star-shaped $\rho$-bounded and ( $\rho$-a.e.)-compact subset of $L_{\rho}$ such that $B-B \subset L_{\rho}^{0}$. Assume in addition that for every sequence of functions $f_{n} \in B$ such that $f_{n} \rightarrow f \rho$-a.e. with $f \in B$ and for every sequence of sets $G_{k} \downarrow \emptyset$,

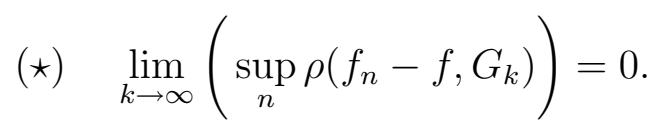


If $T: B \rightarrow B$ is $\rho$-nonexpansive, then it has a fixed point.

\section{Remarks.}

(1) Observe that for some important modular function spaces, e.g. Orlicz and Musielak-orlicz spaces, the fact $B-B \subset L_{\rho}^{0}$ follows from $B$ $\rho$-bounded. Indeed, order continuity of $\rho(f-g,$.$) is in such spaces$ equivalent to $\rho(f-g)<\infty$.

(2) Instead of assuming $\rho$ has Fatou property, we may assume that

$$
\rho(f, H) \leq \liminf _{n \rightarrow \infty} \rho\left(f_{n}, H\right)
$$

for every $H \in \mathcal{P}$ such that $f_{n}$ converges uniformly to $f$ on $H$, where $f_{n}$ and $f$ belong to $L_{\rho}^{0}$. Note that this condition is equivalent to $B_{\rho}(r) \cap L_{\rho}^{0}$ is ( $\rho$-a.e.)-closed in $L_{\rho}^{0}$ for all $r>0$, where $B_{\rho}(r)$ is the $\rho$-ball centered at 0 with radius $r$.

(3) An example of a set $B$ satisfying the condition $(\star)$ such that $B-B \in L_{\rho}^{0}$ is provided by a set $B$ such that $B-B \subset\left\{f \in L_{\rho} ;|f(x)| \leq|g(x)|\right\}$ where $g \in L_{\rho}^{0}$.

As mentionned before, one of the reasons of our interest in $\rho$-behavior of mappings is that the F-norm associated to the function modular is defined in an indirect way and consequetly harder to handle than the function modular. Therefore, one may ask what is the relationship, if there is any, between the F-norm nonexpansiveness and the $\rho$-nonexpansiveness. We have the following partial answer.

Proposition 1. Let $\rho$ be a convex, left-continuous function modular. If

$$
\rho(\lambda(T(f)-T(g))) \leq \rho(\lambda(f-g))
$$

for every $\lambda>0$, then $\|T(f)-T(g)\|_{\rho} \leq\|f-g\|_{\rho}$.

Example. Let $X=(0, \infty)$ and $\Sigma$ be the $\sigma$-algebra of all Lebesgue measurable subsets of $X$. Let $\mathcal{P}$ denote the $\delta$-ring of subsets of finite measure. Define a function modular by

$$
\rho(f)=\frac{1}{e^{2}} \int_{0}^{\infty}|f(x)|^{x+1} d m(x) .
$$


Let $B$ be the set of all measurable functions $f:(0, \infty) \rightarrow \Re$ such that $0 \leq f(x) \leq 1 / 2$. Consider the map

$$
T(f)(x)= \begin{cases}f(x-1), & \text { for } x \geq 1 \\ 0, & \text { for } x \in[0,1] .\end{cases}
$$

Clearly, we have $T(B) \subset B$. For every $f, g \in B$ and $\lambda \leq 1$, we have

$$
\rho(\lambda(T(f)-T(g))) \leq \lambda \rho(\lambda(f-g)),
$$

which implies that $T$ is $\rho$-nonexpansive. On the other hand, if we take $f=1_{[0,1]}$, then

$$
\|T(f)\|_{\rho}>e \geq\|f\|_{\rho}
$$

which clearly implies that $T$ is not $\|.\|_{\rho}$-nonexpansive. Note that $T$ is linear.

Remark on Alspach counterexample. Define the operator

$$
T(f)(x)= \begin{cases}\min \{2,2 f(x)\}, & \text { for } x \in[0,1 / 2] \\ \max \{0,2 f(2 x-1)-2\} & \text { for } x \in(1 / 2,1]\end{cases}
$$

on $C$, a convex subset of $L_{1}[0,1]$, defined by

$$
C=\left\{f \in L_{1}[0,1] ; 0 \leq f(x) \leq 2 \text { a.e. and } \int_{0}^{1} f(x) d x=1\right\} .
$$

The operator $T$ is an isometry on $C$, with an empty fixed point set. It seems that the condition $\int_{0}^{1} f(x) d x=1$ is "responsible" for the nonexistence of a fixed point. Note that Theorem 3 gives "an intrinsic" reason why $T$, defined on $B=\left\{f \in L_{1}[0,1] ; 0 \leq f(x) \leq 2\right.$ a.e. $\}$, must have a fixed point while $T: C \rightarrow C$ does not have to. Moreover, we do not refer to any geometrical properties of subspaces of $L_{1}$ (observe that even the convexity of $B$ is not essential here).

In the next section we will discuss the concept of normal structure properties in the modular sense and state Kirk's fixed point theorem. 


\section{Normal structure in modular spaces}

The concept of normal structure was introduced by Brodskii and Milmann for the case of linear normed spaces. Kirk was the first to link this concept to existence of fixed point of nonexpansive mappings. There were some attempts to generalize the concept of normal structure to metric spaces and more abstract sets. In this section we define normal structure for function modulars.

Definition 11. Let $B$ be a $\rho$-bounded subset of $L_{\rho}$.

(a) By the $\rho$-diameter of $B$, we will understand the number

$$
\delta_{\rho}(B)=\sup \{\rho(f-g) ; f, g \in B\} .
$$

(b) The quantity $r_{\rho}(f, B)=\sup \{\rho(f-g) ; g \in B\}$ will be called the $\rho$ Chebyshev radius of $B$ with respect to $f$.

(c) The $\rho$-Chebyshev radius of $B$ is defined by $R_{\rho}(B)=\inf \left\{r_{\rho}(f, B) ; f \in\right.$ $B\}$.

(d) The $\rho$-Chebyshev center of $B$ is defined as the set

$$
\mathcal{C}_{\rho}(B)=\left\{f \in B ; r_{\rho}(f, B)=R_{\rho}(B)\right\}
$$

Note that $R_{\rho}(B) \leq r_{\rho}(f, B) \leq \delta_{\rho}(B)$ for all $f \in B$ and observe that there is no reason, in general, for $\mathcal{C}_{\rho}(B)$ to be nonempty.

Definition 12. Let $B$ be a $\rho$-bounded subset of $L_{\rho}$.

(a) We say that $f$ is a $\rho$-diametral point of $B$ if $r_{\rho}(f, B)=\delta_{\rho}(B)$.

(b) The set $B$ is called $\rho$-diametral if every $f \in B$ is a $\rho$-diametral point of $B$.

(c) A sequence $\left\{f_{n}\right\}$ from $L_{\rho}$ is called a $\rho$-diametral sequence if there exists $c>0$ such that $\delta_{\rho}\left(f_{n}\right) \leq c$ and

$$
\lim _{n \rightarrow \infty} \operatorname{dist}_{\rho}\left(f_{n+1}, \operatorname{conv}\left(f_{1}, . ., f_{n}\right)\right)=c .
$$


where $\operatorname{dist}_{\rho}(f, A)=\inf \{\rho(f-g) ; g \in A\}$ and

$$
\operatorname{conv}\left(f_{1}, . ., f_{n}\right)=\left\{\sum_{i=1}^{n} \alpha_{i} f_{i} ; \alpha_{i} \geq 0 \text { and } \sum_{i} \alpha_{i}=1\right\} .
$$

Let us observe that $\operatorname{dist}_{\rho}\left(f_{n+1}, \operatorname{conv}\left(f_{1}, . ., f_{n}\right)\right) \leq n c$, while in the norm case this distance can be estimated by the number $c$ itself.

Definition 13. Let $B$ be a $\rho$-bounded subset of $L_{\rho}$.

(a) We say that $A$ is an admissible subset of $B$ if

$$
A=\bigcap_{i \in I} B_{\rho}\left(b_{i}, r_{i}\right) \cap B,
$$

where $b_{i} \in B, r_{i} \geq 0$ and $I$ is an arbitrary index set.

(b) If $C$ is a subset of $B$, we let

$$
\operatorname{co}(C)=\bigcap_{f \in C} B_{\rho}\left(f, r_{\rho}(f, C)\right) \cap B .
$$

(c) $B$ is said to have $\rho$-normal structure property if each $\rho$-admissible subset $A$ of $B$, not reduced to a single point, has a point which is not $\rho$-diametral.

By $\mathcal{A}(B)$ we denote the family of all admissible subsets of $B$. Note that if $B$ is $\rho$-bounded, then $B \in \mathcal{A}(B)$.

The classical proof of Kirk's fixed point theorem relies heavily on a topological compactness assumption. Under our formulation, we may assume a sequential compactness which is easy to check in many practical cases. Therefore we may ask when our sequential compactness is generated from a topological compactness,i.e. given a set which is sequentially compact (in our sense), is there a topology which makes it compact? This problem to our knowledge is still open. Consequently, we will use a "constructif proof" developped by Kirk instead of the classical proof based on Zorn and the compactness assumption. It is worth to mention that Kirk's fixed point theorem in modular spaces is the first example where the constructif proof was used. 
The main ingredient in the constructif proof is the following technical result.

Lemma 1. Let $B$ be a nonempty, $\rho$-bounded subset of $L_{\rho}$. Let $T: B \rightarrow B$ be a $\rho$-nonexpansive mapping. Assume that $B$ has $\rho$-normal structure. If $D \in \mathcal{A}(B)$ is $T$-invariant, i.e. $T(D) \subset D$, then there exists $D^{\star} \in \mathcal{A}(B)$ a nonempty subset of $D$, which is $T$-invariant, and such that

$$
\delta_{\rho}\left(D^{\star}\right) \leq \frac{1}{2}\left(\delta_{\rho}(D)+R_{\rho}(D)\right) .
$$

We are now ready to state the analog of Kirk's fixed point theorem in modular function spaces.

Theorem 4. Let $\rho$ have the Fatou property and $B$ be a nonempty, $\rho$-bounded subset of $L_{\rho}$. Assume that $B$ has $\rho$-normal structure and is ( $\rho$-a.e.)-compact. If $T: B \rightarrow B$ is $\rho$-nonexpansive, then it has a fixed point.

The analog of Kirk's fixed point theorem for commutative families is also true in modular spaces.

Theorem 5. Let $\rho$ have the Fatou property and $\mu$ be a positive $\sigma$-finite measure which is absolutely continuous with respect to $\rho$. Let $B$ be a nonempty, $\rho$-bounded subset of $L_{\rho}$ which is compact in the sense of convergence in measure on sets of finite measure. Assume that $B$ has $\rho$-normal structure. Then any commutative family of $\rho$-nonexpansive self-mappings of $B$ has a common fixed point.

Recall that a positive $\sigma$-finite measure $\mu$ on $\Sigma$ is said to be absolutely continuous with respect to the function modular $\rho$ if $\mu(A)=0$ for any $\rho$-null set $A$ (recall that $A$ is $\rho$-null if $\rho\left(\alpha 1_{A}\right)=0$ for all $\alpha>0$ ).

We will not comment on the last two theorems since many authors have written extensively on them. Let us only mention that one of the reasons why they were at the center of the metric fixed point theory is du to the rich structure of normal structure property in Banach spaces. Therefore, it is normal to investigate $\rho$-normal structure in modular spaces. Traditionally, the first results in this direction connect normal structure to geometrical properties like uniform convexity. Generalizations of these notions to metric spaces 
have been known for a while but no concrete example was known until the hyperbolic metric on the Hilbert ball was proven to be uniformly convex. In what follows we give the definition of uniform convexity of function modulars.

\section{Definition 14.}

(a) For nonzero $f \in L_{\rho}$ and $r>0$, we define the $r$-modulus of uniform convexity of $\rho$ in the direction of $f$ to be:

$$
\delta_{\rho}(r, f)=\inf \left\{1-\frac{1}{r} \rho\left(g+\frac{1}{2} f\right)\right\},
$$

where the infinimum is taken over all $g \in L_{\rho}$ such that $\rho(g) \leq r$ and $\rho(g+f) \leq r$.

(b) We say that a convex function modular $\rho$ is uniformly convex in every direction (UCED) if $\delta_{\rho}(r, f)>0$ for every nonzero $f \in L_{\rho}$ and $r>0$.

(c) We say that a convex function modular $\rho$ is uniformly convex (UC) if for every $\varepsilon>0$ and $r>0$, we have

$$
\delta_{\rho}(r, \varepsilon)=\inf \left\{\delta_{\rho}(r, f): f \in L_{\rho} \text { and } \rho(f) \geq r \varepsilon\right\}>0 .
$$

Note that the definition of uniform convexity for function modulars given by Musielak is the exact translation of the known one in normed linear spaces. Such a concept does not seem to be an appropriate tool for dealing with modulars without the $\Delta_{2}$-condition, which is of particular interest to us (see example below).

Remark. Let us observe that $\delta_{\rho}(r, \varepsilon)$ is an increasing function of $\varepsilon$ for every fixed $r$. Moreover, for $r_{1}<r_{2}$, there holds

$$
1-\frac{r_{2}}{r_{1}}\left(1-\delta_{\rho}\left(r_{2}, \varepsilon \frac{r_{1}}{r_{2}}\right)\right) \leq \delta_{\rho}\left(r_{1}, \varepsilon\right) .
$$

Let us also mention that, since $\rho$ does not have to be homogenuous, $\delta_{\rho}(r, f)$ depends on $r$ not only the direction $f$. 
The classical link between these notions and $\rho$-normal structure is given in the following result.

Proposition 2. Let $\rho$ be a UCED function modular, and let $B \subset L_{\rho}$ be starshaped, $\rho$-bounded and not reduced to one point. Then $B$ has a point which is not $\rho$-diametral.

Nontrivial examples of function modulars which are uniformly convex are to be found in Orlicz spaces. Therefore, we will restrict ourselves till the end of this section to Orlicz modulars. We will assume that $\mu$ is finite and atomless. However, by simple modification of proofs all our results can be obtained in the $\sigma$-finite case.

\section{Definition 15.}

(a) An Orlicz-function $\varphi$ is said to be strictly convex (SC) if and only if for every $x \neq y$ there holds

$$
\varphi\left(\frac{x+y}{2}\right)<\frac{\varphi(x)+\varphi(y)}{2} .
$$

(b) An Orlicz-modular $\rho$ is said to be strictly convex (SC) if and only if for every $f, g \in L_{\rho}$ such that $\rho(f)=\rho(g)$ and

$$
\rho\left(\frac{f+g}{2}\right)=\frac{\rho(f)+\rho(g)}{2}
$$

there holds $f=g$.

(c) An Orlicz-function $\varphi$ is said to be uniformly convex (UC) if and only if for every $a \in(0,1)$, there exists $\delta(a) \in(0,1)$ such that

$$
\varphi\left(\frac{1+b}{2}\right)<(1-\delta(a)) \frac{\varphi(x)+\varphi(b x)}{2}
$$

for every $x>0$ and $0 \leq b \leq a$.

Note that Kaminska proved that for the norm $\|.\|_{\rho}$ the following assertions are equivalent:(a) $\|.\|_{\rho}$ is $\mathrm{UCED}$, (b) $\|.\|_{\rho}$ is $\mathrm{SC}$, (c) $\varphi$ is $\mathrm{SC}$ and $\varphi$ satsifies $\Delta_{2}$. A similar result for uniform convexity was also proved by Kaminska. 
Our next results give their modular version. Note, however, that when replacing $\|.\|_{\rho}$ by $\rho$, we were able to eliminate the $\Delta_{2}$-condition.

Theorem 6. The following three conditions are equivalent:

(i) $\varphi$ is $S C$;

(ii) $\rho$ is $S C$;

(iii) $\rho$ is UCED.

Definition 16. A function $\varphi$ is called very convex (VC) if and only if for every $\varepsilon>0$ and any $x_{0}>0$, there exists $\delta>0$ such that

$$
\varphi\left(\frac{1}{2}(x-y)\right) \geq \frac{\varepsilon}{2}(\varphi(x)+\varphi(y)) \geq \varepsilon \varphi\left(x_{0}\right)
$$

implies

$$
\varphi\left(\frac{1}{2}(x+y)\right) \leq \frac{1}{2}(1-\delta)(\varphi(x)+\varphi(y)) .
$$

It is easy to see that $\varphi$ is $\mathrm{VC}$ if $\varphi$ is UC. The characterization of uniformly convex Orlicz modulars is given in the following theorem.

Theorem 7. The following conditions are equivalent:

(i) $\varphi$ is $V C$;

(ii) $\rho$ is $U C$.

The proof is based on the following technical result.

Lemma 2. Let $\varphi$ be very convex. To every $\varepsilon>0, s>0$ there exists $\eta>0$ (which depends only on $\varepsilon$ and $s$ ) such that $\delta_{\rho}(r, \varepsilon)>\eta$ for $r>s / 3$.

The functions $\varphi_{1}(t)=e^{|t|}-|t|-1$ and $\varphi_{2}(t)=e^{t^{2}}-1$ may serve as examples of very convex Orlicz-functions which do not satisfy $\Delta_{2}$-condition. Nevertheless, by the previuous theorems, we can obtain information about the associate function modular and about existence of fixed points of $\rho$-nonexpansive mappings. The associated Luxemburg-norm fails to be nice in this case du to Kaminska 's results. Therefore we cannot use the classical theorems even when the mappings are norm-nonexpansive. Let us add before we end this 
section, that Lami-Dozo and Turpin obtained a similar fixed point theorem for $\rho$-nonexpansive mappings for Musielak-Orlicz spaces. Instead of strict convexity of $\varphi$ they assumed some growth condition and $B$ was assumed to satisfy a stronger kind of $\rho$-boundedness $(\sup \{\rho(\lambda(f-g)) ; f, g \in B\}<\infty$ for some $\lambda>1$ ).

The last result of this section is an analog of Garkavi's characterization of UCED in normed linear spaces.

Theorem 8. The following conditions are euivalent:

(i) $\rho$ is UCED;

(ii) For every nonempty subset $B$ of $L_{\rho}, \rho$-bounded, $\rho$-closed and convex, we have $\mathcal{C}_{\rho}(B)$ has at most one point.

\section{Uniform noncompact convexity in Modular spaces.}

Goebel and Sekowski used the concept of measure of noncompactness to give a new classification of Banach spaces. In this work, we discuss their result in modular spaces.

Definition 17. Let $X_{\rho}$ be a modular space. Define the Hausdorff measure of noncompactness by

$\chi(A)=\inf \{\varepsilon>0 ; A$ can be covered with a finite number of $\rho$-balls of radius less than $\varepsilon\}$,

and the Kuratowski measure of noncompactness by

$\alpha(A)=\inf \{\varepsilon>0 ; A$ can be covered with a finite number of sets of $\rho$-diameter less than $\varepsilon\}$,

for any subset $A$ of $X_{\rho}$. We will make the obvious convention that the inf over empty set is infinite.

One can easily notice that $\chi(A) \leq \alpha(A)$ for every $A \subset X_{\rho}$. 
Throughout this work, we will assume that $X_{\rho}$ is $\rho$-complete and $\rho$ satisfies the Fatou property.

Proposition 3. The following properties hold,

(1) if $A \subset B$, then $\chi(A) \leq \chi(B)$ and $\alpha(A) \leq \alpha(B)$,

(2) $\chi(\bar{A})=\chi(A)$ and $\alpha(\bar{A})=\alpha(A)$,

(3) if $\alpha(A)=0$, then $\bar{A}$ is $\rho$-compact,

(4) let $\left(A_{n}\right)$ be a decreasing sequence of nonempty $\rho$-closed subset of $X_{\rho}$. Assume that $\lim _{n} \alpha\left(A_{n}\right)=0$ (resp. $\lim _{n} \chi\left(A_{n}\right)=0$ ) then $\cap A_{n}$ is a nonempty $\rho$-compact set (resp. $\cap A_{n}$ is nonempty and $\chi\left(\cap A_{n}\right)=0$ ).

Since $\rho$ lakes a priori the subadditivity, there is no reason to have $\alpha(A)=0$ whenever $A$ is $\rho$-compact. Let us add that it can be shown that $\rho$ satisfies the $\Delta_{2}$-condition if and only if $\alpha(A)=0$ whenever $A$ is $\rho$-compact.

As Goebel and Sekowski did in Banach spaces, we give a new scaling for modular spaces using the measures of noncompactness $\alpha$ and $\chi$.

Definition 18. The $\rho$-modulus of noncompact convexity $\Delta_{\chi}\left(\right.$ resp. $\left.\Delta_{\alpha}\right)$ is defined as

(**) $\Delta_{\chi}(r, \varepsilon)=\sup \left\{c>0 ;\right.$ for any $A \subset X_{\rho} \rho$-bounded convex such that $A \subset B_{\rho}(f, r)$

$$
\text { with } \left.\chi(A) \geq r \varepsilon, \text { then } \operatorname{dist}_{\rho}(f, A) \leq r(1-c)\right\},
$$

for every $r>0$ and $\varepsilon>0$.

For the definition of $\Delta_{\alpha}$, one will replace $\chi(A)$ by $\alpha(A)$ in $(* *)$.

Define the characteristic of noncompact convexity by

$$
\varepsilon_{\chi}\left(r, X_{\rho}\right)\left(\text { resp. } \varepsilon_{\alpha}\left(r, X_{\rho}\right)\right)=\sup \left\{\varepsilon>0 ; \Delta_{\chi}(r, \varepsilon)\left(\text { resp. } \Delta_{\alpha}(r, \varepsilon)\right)=0\right\}
$$

for every $r>0$.

Since we have $\chi \leq \alpha$, one can get $\Delta_{\chi} \leq \Delta_{\alpha}$ and $\varepsilon_{\alpha} \leq \varepsilon_{\chi}$. In any Banach space $X$, one can easily prove that $\varepsilon_{\chi}(X)=0$ if and only if $\varepsilon_{\alpha}(X)=0$. In 
modular spaces, it is not the case in general.

Definition 19. The modular space $X_{\rho}$ is said to be $\alpha$ (resp. $\chi$ )-uniformly $\rho$-noncompact convex if and only if $\varepsilon_{\alpha}\left(r, X_{\rho}\right)=0\left(\operatorname{resp} . \varepsilon_{\chi}\left(r, X_{\rho}\right)=0\right)$, for every $r>0$.

Clearly if $X_{\rho}$ is $\chi$-uniformly $\rho$-noncompact convex, then $X_{\rho}$ is $\alpha$-uniformly $\rho$-noncompact convex.

Example. It is not hard to show that

$$
\left.\delta_{\rho}(r, \zeta \varepsilon)\right) \leq \Delta_{\alpha}(r, \varepsilon),
$$

for every $r>0, \varepsilon>0$ and $\zeta<1$, where $\delta_{\rho}$ is the $\rho$-modulus of uniform convexity. Clearly, we have $\rho$-uniform convexity implies $\alpha$-uniform $\rho$ noncompact convexity.

Goebel and Sekowski proved that whenever the characteristic of uniform noncompact convexity of any Banach space is less than 1, then the space is reflexive and has the normal structure property. In what follows, we investigate the validity of these results in modular spaces. Let us point out that their proofs are entirely based on the rich structure of the Banach spaces, specially the existence of the dual space.

The first result discuss the link between proximinality and the $\rho$-modulus of noncompact convexity. More exactly, given a function $f \in X_{\rho}$, we consider the minimization problem of finding $h \in C$ such that

$$
\rho(f-h)=\inf \{\rho(f-g) ; g \in C\},
$$

for a given $C \subset X_{\rho}$. Such a $h$ is called a best approximant. Problems of finding best approximants are important in approximation theory and probability theory.

Theorem 9. Let $X_{\rho}$ be a $\rho$-complete modular space. Assume that $\rho$ is convex, satisfy the Fatou property and $X_{\rho}$ is $\alpha$-uniformly $\rho$-noncompact convex. Then for any nonempty $C \rho$-bounded $\rho$-closed convex subset of $X_{\rho}$ and $f \in X_{\rho}$ such that $\operatorname{dist}_{\rho}(f, C)<\infty$, the set

$$
P_{\rho}(f, C)=\left\{g \in C ; \operatorname{dist}_{\rho}(f, C)=\rho(f-g)\right\}
$$


is a nonempty $\rho$-compact convex subset.

Remark. We are unable to prove whether the conclusion of Theorem 9 is true if we juste assume that $\varepsilon_{\alpha}\left(X_{\rho}\right)<1$.

Theorem 10. Let $X_{\rho}$ be a $\rho$-complete modular space. Assume that $\rho$ is convex, satisfy the Fatou property and $X_{\rho}$ is $\alpha$-uniformly $\rho$-noncompact convex. Then $X_{\rho}$ has the Property $(R)$.

The definition of property $(R)$ is given in Definition 6 .

Remark. One can ask if $\bigcap_{\beta \in \Gamma} A_{\beta}$ is nonempty under the assumptions of Theorem 10, for any decreasing family $\left(A_{\beta}\right)_{\beta \in \Gamma}$ of $\rho$-bounded, $\rho$-closed nonempty convex subsets of $X_{\rho}$ and any directed set $\Gamma$. The answer to this problem is in the affirmative. Consequently, the proof of Kirk's fixed point theorem in the modular sense will use the classical ideas instead of the contructive ones.

The next result discuss the $\rho$-normal structure in uniformly $\rho$-noncompact convex modular spaces.

Theorem 11. Let $X_{\rho}$ be a $\rho$-complete modular space. Assume that $\rho$ is convex, satifies the Fatou property and $\varepsilon_{\alpha}\left(X_{\rho}\right)<1$. Then $X_{\rho}$ has the $\rho$-normal structure provided that $X_{\rho}$ has the property $\left(R^{\prime}\right)$.

\section{Opial and Kadec-Klee properties in modu- lar spaces}

In this section, we discuss some recent results (see Besbes and Lennard) on uniform Kadec-Klee and uniform Opial properties in $L^{1}$. It is not very hard to see that these results are of measure theoretical nature. It is worth to mention that the original ideas are to be found in Brezis and Lieb's work as was noticed by Lami-Dozo and Turpin.

After close investigation, it was clear to us that these results are not of metric nature and can be translated into modular sense. Note that in $L^{1}$, the norm and the modular coincide. 
Throughout this section, $\rho$ is assumed to be convex. Before we give the main result, we need the analog of Brezis and Lieb's result for function modulars.

Lemma 3. Let $\varepsilon>0$ and $k>1$ be such that $k \varepsilon<1$. Set $C_{\varepsilon}=1 / \varepsilon(k-1)$. Then for every $f, g \in L_{\rho}$ such that $\rho(k f)<\infty$ and $\rho\left(C_{\varepsilon} g\right)<\infty$, we have

$$
|\rho(f+g)-\rho(f)| \leq \varepsilon|\rho(k f)-k \rho(f)|+2 \rho\left(C_{\varepsilon} g\right) .
$$

From now on we will assume that $\rho$ is additive, i.e.

$$
\rho(f, A \cup B)=\rho(f, A)+\rho(f, B),
$$

whenever $A, B \in \Sigma$ such that $A \cap B=\emptyset$. Clearly this implies $\rho(f, A)=$ $\rho\left(f 1_{A}\right)$.

The next result states the main result of this work.

Theorem 12. Let $\left\{f_{n}\right\} \in L_{\rho}$ be $\rho$-a.e. convergent to 0 . Assume there exists $k>1$ such that

$$
\sup _{n} \rho\left(k f_{n}\right)=M<\infty .
$$

Let $g \in E_{\rho}$, then we have

$$
\liminf _{n \rightarrow \infty} \rho\left(f_{n}+g\right)=\liminf _{n \rightarrow \infty} \rho\left(f_{n}\right)+\rho(g) .
$$

As a corllary to this theorem one can get the following.

Corollary 1. Let $p \geq 1$ and $\left\{f_{n}\right\}$ be a sequence of $L^{p}$-uniformly bounded functions on a measure space. Assume that $\left\{f_{n}\right\}$ converges almost everywhere to $f \in L^{p}$. Then

$$
\liminf _{n \rightarrow \infty}\left\|f_{n}\right\|^{p}=\liminf _{n \rightarrow \infty}\left\|f_{n}-f\right\|^{p}+\|f\|^{p} .
$$

When $p=1$, the conclusion of Corollary 1 gives the main result of Besbes and Lennard. Let us add that when $p<1$ the conclusion of Corollary 1 is 
still true. This will not be a simple deduction from Theorem 12 since the function $\varphi(t)=t^{p}$ is not convex. A technical assumption can be added to get a more general result.

Theorem 13. Let $\varepsilon>0$ and $\left\{f_{n}\right\} \in L_{\rho}$ be $\rho$-a.e. convergent to 0 . Assume there exists $k>1$ such that

$$
\sup _{n} \rho\left(k f_{n}\right)=M<\infty .
$$

Let $f \in E_{\rho}$ such that $\rho(f) \geq \varepsilon$, then we have

$$
\liminf _{n \rightarrow \infty} \rho\left(f_{n}\right)+\varepsilon \geq \liminf _{n \rightarrow \infty} \rho\left(f_{n}+f\right) .
$$

The proof is obvious using the conclusion of Theorem 12. This is a kind of Opial property. First let us give the following definition.

Definition 20. We will say that $L_{\rho}$ satisfies the $\rho$-a.e.-Opial property if for every $\left\{f_{n}\right\} \in L_{\rho} \rho$-a.e. convergent to 0 such that there exists $k>1$ for which

$$
\sup _{n} \rho\left(k f_{n}\right)=M<\infty
$$

then for every $f \in E_{\rho}$ not equal to 0 we have

$$
\liminf _{n \rightarrow \infty} \rho\left(f_{n}\right)<\liminf _{n \rightarrow \infty} \rho\left(f_{n}+f\right) .
$$

We will say that $L_{\rho}$ satisfies the $\rho$-a.e.-uniform Opial property if for every $\varepsilon>0$ there exists $\eta>0$ such that for every $\left\{f_{n}\right\} \in L_{\rho} \rho$-a.e. convergent to 0 such that there exists $k>1$ for which

$$
\sup _{n} \rho\left(k f_{n}\right)=M<\infty
$$

then for every $f \in E_{\rho}$ such that $\rho(f) \geq \varepsilon$ we have

$$
\liminf _{n \rightarrow \infty} \rho\left(f_{n}\right)+\eta \leq \liminf _{n \rightarrow \infty} \rho\left(f_{n}+f\right) .
$$

Opial's property plays an important role in the study of convergence of iterates of nonexpansive mappings and of the asymptotic behavior of nonlinear semigroups. Clearly the $\rho$-a.e.-uniform Opial property implies $\rho$-a.e.-Opial 
property. Therefore the conclusion of Theorem 13 means that $L_{\rho}$ satisfies the $\rho$-a.e.-uniform Opial property.

Definition 21. We will say that $L_{\rho}$ satisfies $\rho$-a.e.-Kadec-Klee property if for some $\varepsilon>0$ and every $r>0$ there exists $\eta>0$ such that for every $\left\{f_{n}\right\} \in E_{\rho}$ $\rho$-a.e. convergent to $f \in E_{\rho}$ such that there exists $k>1$ for which

$$
\sup _{n} \rho\left(k\left[f_{n}-f\right]\right)=M<\infty
$$

and $\rho\left(f_{n}\right) \leq r$ for every $n \geq 1$, we have

$$
\rho(f) \leq r(1-\eta)
$$

provided

$$
\operatorname{sep}\left\{\frac{1}{2} f_{n}\right\}=\inf \left\{\rho\left(\frac{f_{n}-f_{m}}{2}\right) ; n \neq m\right\}>r \varepsilon .
$$

We will say that $L_{\rho}$ satisfies $\rho$-a.e.-uniform Kadec-Klee property if the above still holds for every $\varepsilon$.

Theorem 14. Under the assymptions of Theorem 12, the modular function space $L_{\rho}$ satisfies $\rho$-a.e.-uniform Kadec-Klee property.

If the modular $\rho$ is subadditive then one does not need to take $\rho\left(\left(f_{n}-\right.\right.$ $\left.f_{m}\right) / 2$ ) in Definition 22 we could take $\rho\left(f_{n}-f_{m}\right)$. This is the case when $L_{\rho}=L^{1}$.

\section{Semigroups in Musielak-Orlicz spaces.}

In this section, we consider the classical Musielak-Orlicz spaces $L^{\varphi}$, in which we investigate the existence and the behavior of nonlinear semigroups. We obtain an existence result of semigroups generated by the mapping $A=I-T$, where $T$ is $\rho$-nonexpansive acting within $L^{\varphi}$. Note that $\rho$ does not have to satisfy the $\Delta_{2}$-condition.

Let us also add that the classical approch consists of solving an initial value problem. When $\rho$ satisfies the $\Delta_{2}$-condition, our existence result seems to be unknown. 
Definition 22. Let $C$ be a subset of $L^{\varphi}$. A mapping $S:[0, \infty) \times C \rightarrow C$ is said to be a ( $\rho$-nonexpansive)-semigroup if the following conditions are satisfied

(i) $S(0) f=f$ for all $f \in C$,

(ii) $S\left(t_{1}+t_{2}\right)=S\left(t_{1}\right) S\left(t_{2}\right)$ for all $t_{1}, t_{2} \geq 0$,

( (iii) the mapping $f \rightarrow S(t) f$ is $\rho$-nonexpansive for all $t \geq 0$ ).

In order to obtain an existence result concerning the semigroups in MusielakOrlicz spaces, the following technical theorem is needed.

Theorem 15. Let $C$ be a $\rho$-closed, $\rho$-bounded convex subset of $L^{\varphi}$. Let $T$ : $C \rightarrow C$ be $\rho$-nonexpansive and norm-continuous. Let $f \in C$ be fixed and consider the recurrent sequence defined by

$$
\left\{\begin{array}{l}
u_{0}(t)=f \\
u_{n+1}(t)=\exp (-t) f+\int_{0}^{t} \exp (s-t) T\left(u_{n}(s)\right) d s
\end{array}\right.
$$

for $t \in[0, A]$, where $A$ is a fixed positive number. Then the sequence $\left\{u_{n}(t)\right\}$ is $\rho$-Cauchy for any $t \in[0, A]$. Therefore it converges with respect to $\rho$, to $u(t) \in C$ for any $t \in[0, A]$.

The proof of Theorem 15 is based on the following technical lemma.

Lemma 4. Let $x, y:[0, t] \rightarrow L^{\varphi}$ be norm-continuous mappings. Then, we have

$\rho\left(\exp (-t) y(t)+\int_{0}^{t} \exp (s-t) x(s) d s\right) \leq \exp (-t) \rho(y(t))+K(t) \sup \{\rho(x(s)) ; s \in[0, t]\}$

where $K(t)=1-\exp (-t)=\int_{0}^{t} \exp (s-t) d s$.

It is not clear if the assumptions on $C$ and $T$ are enough to imply any good behavior of $u(t)$ on $[0, A]$ such as norm-continuity for example. But if $\rho$ satisfies the $\Delta_{2}$-condition then $u(t)$ is indeed continuous.

Theorem 16. Under the assumptions of Theorem 2.1., if moreover $\rho$ satisfies the $\Delta_{2}$-condition, then $u(t)$ is solution of the following initial value 
problem,

$$
\left\{\begin{array}{l}
u^{\prime}(t)+(I-T) u(t)=0 \\
u(0)=f .
\end{array}\right.
$$

Remark. Notice that when $\rho$ satisfies the $\Delta_{2}$-condition there is no reason for $T$ to be norm-nonexpansive. So the classical theorems related to the existence of solutions to the initial value problem won't apply.

Remark. Let $L>A$ and consider the following system

$$
\left\{\begin{array}{l}
U_{0}(t)=f \\
U_{n+1}(t)=\exp (-t) f+\int_{0}^{t} \exp (s-t) T U_{n}(s) d s
\end{array}\right.
$$

for $t \in[0, L]$. Then $\left\{U_{n}(t)\right\}$ is $\rho$-convergent to $U(t)$ and $U(t)=u(t)$ for $t \in[0, A]$. This implies that there exists $u(t) \in C$ for all $t \in[0, \infty)$, such that the restriction of $u$ to $[0, A]$ is the $\rho$-limit of the sequence $\left\{u_{n}(t)\right\}$ given in Theorem 15. We will use the notation $u_{f}$ to designate this function $u$ associated to the initial condition $u(0)=f$.

In the next result we discuss the existence of $\rho$-nonexpansive semigroups in $L^{\varphi}$.

Theorem 17. Let $C$ and $T$ be as stated in Theorem 15. For any $f \in C$ consider $u_{f}(t) \in C$ for $t \in[0, \infty)$. Define $S:[0, \infty) \times C \rightarrow C$ by

$$
S(t) f=u_{f}(t) .
$$

Then $S$ defines a $\rho$-nonexpansive semigroup.

We conclude this section by a result which links the set of fixed points of the semigroup $S$ and the set of fixed points of $T$.

Proposition 4. Define the set $F(S)$ to be the set of $f \in C$ such that $S(t) f=$ f for all $t \geq 0$. Then we have

$$
F(S)=F(T) .
$$




\section{References}

[1] D. Alspach, "A fixed point free nonexpansive mapping", Proc. Am. Mat. Soc. 82(1981), 423-424.

[2] A.G. Aksoy, M. A. Khamsi, "Nonstandard Methods in fixed point theory", Springer-Verlag, New York and Berlin, 1990.

[3] J. Banas and K. Goebel, "Measures of noncompactness in Banach spaces", Lecture Notes in Pure and Applied Math., Marcel Dekker, Vol. 60, New York, 1980.

[4] M. Besbes, "Points fixes des contractions definies sur un convexe $L^{0}$ ferme de $L^{1}$ ", C.R.A. Sc. de Paris, Tome 311, Serie I (1990), 243-246.

[5] Z. Birnbaum, W. Orlicz, "Uber die Verallgemeinerung des Begriffes der zueinander konjugierten Potenzen", Studia Math. 3(1931), 1-67.

[6] H. Brezis, E. Lieb, "A relation between pointwise convergence of functions and convergence of functionals", Proc. A.M.S. Vol. 88-3(1983), 486-490.

[7] M.S. Brodskii and D.P. Milman, "On the center of a convex set", Dokl. Acad. Nauk. SSSR 59 (1948), 837-840.

[8] F.E. Browder, "Nonexpansive nonlinear operators in a Banach space", Proc. Nat. Acad. Sci. USA. 54(1965), 1041-1044.

[9] S. Chen, M.A. Khamsi and W.M. Kozlowski, "Some geometrical properties and fixed point theorems in Orlicz modular spaces", Journal of Mathematical Analysis and Applications, Vol. 155, No.2 (1991), 393412.

[10] M.C. Crandall, A. Pazy, "Semigroups of nonlinear contractions and dissipative sets", Jour. Funct. Anal., Vol.3(1963), 376-418.

[11] D. van Dulst, B. Sims, "Fixed points of nonexpansive mappings and Chebyshev centers in Banach spaces with norms of type (KK)", Banach space theory and its applications, Proc. Bucharest 1981, Lecture Notes in Math. 991, Springer, 1983, 35-43. 
[12] D. van Dulst, V. de Valk, "(KK)-properties, normal structure and fixed points of nonexpansive mappings in Orlicz spaces", Canad. J. Math. 38 (1986), 728-750.

[13] K. Goebel, W.A. Kirk, "Topics in Metric fixed point theory", Cambridge University Press, Cambridge, 1990.

[14] K. Goebel and S. Reich, " Uniform convexity, and Nonexpansive mappings", Dekker, New York 1984.

[15] K. Goebel and T. Sekowski, " The modulus of noncompact convexity", Ann. Univ. Mariae Curie-Sklodowska 38, Sect. A(1984).

[16] K. Goebel, T. Sekowski and A. Stachura, "Uniform convexity of the Hyperbolic metric and fixed points of holomorphic mappings in the Hilbert ball", Nonlinear Analysis 4 (1980), 1011-1021.

[17] D. Gohde, "Zum Pinzip der kontraktiven abbildung", Math. Nachr. 30(1965), 251-258.

[18] J. Gorniki, "Some remarks on almost convergence of the Picard iterates for nonexpansive mappings in Banach spaces which satisfy the Opialcondition", Comment. Math. 29(1988),59-68.

[19] H. Hudzik, A. Kaminska and J. Musielak, "On the convexity coefficient of Orlicz spaces", math. Z. 197(1988), 291-295.

[20] R. Huff, "Banach spaces which are nearly uniformly convex", Rocky Mountain J. Math. 10 (1980), 743-749.

[21] V.I. Istratescu, J.R. Partington, "On nearly uniformly convex and kuniformly convex spaces", Math. Proc. Cambridge Philo. Soc. 95(1984), 325-327.

[22] A. Kaminska, "On uniform convexity of Orlicz spaces", Indag. Math. 44, No.1(1982), 27-36.

[23] A. Kaminska, "On some convexity properties of Musielak-Orlicz spaces", Supplemento ai Rend. Circ. Mat. Palermo (2) Suppl. 5(1984), 63-72.

[24] L. Karlovitz, "On nonexpansive mappings", Proc. A.M.S. 55(1976), 321325. 
[25] M.A. Khamsi, W.M. Kozlowski, S. Reich, "Fixed point theory in Modular function spaces", Nonlinear Analysis Th. M. Appl., Vol. 14-11(1990), 935-953.

[26] W.A. Kirk, "A fixed point theorem for mappings which do not increase distances", Amer. Math. Monthly 71(1965), 1004-1006.

[27] W.A. Kirk, "Nonexpansive mappings in metric and Banach spaces", Estratto Dai (Rend. Sem. Mat. Fis. Milano) 51 (1981), 133-144.

[28] M.A. Krasnoselskii, Y.B. Rutickii, "Convex functions and Orlicz spaces", (translation), Noordhoff, groningen, 1961.

[29] W.M. Kozlowski, “ Modular function spaces", Dekker, New York, Basel 1988.

[30] T. Kuczumow, "Weak convergence theorems for nonexpansive mappings and semi-groups in Banach spaces with Opial' s property", Proc. Amer. Math. Soc. 93 (1985), 430-432.

[31] E. Lami-Dozo, Ph. Turpin, "Nonexpansive maps in generalized Orlicz spaces", Studia Math. 8691987), 155-188.

[32] C. Lennard, "A new convexity property that implies a fixed point property for $L_{1}$ ", Studia Math. 100-2(1991), 95-108.

[33] W.A.J. Luxemburg, "Banach function spaces", Thesis, Delft 1955.

[34] W.A.J. Luxemburg, A.C. Zaanen, "Notes on Banach function spaces I-XII", Proc. Acad. Sci. Amsterdam, (1963) A-66, 135-153, 239-263-, 496-504, 655-681, (1964) A-64, 101-119, (1964) A-67, 360-376, 493-543.

[35] J. Musielak, "Orlicz spaces and Modular spaces", Lecture Notes in Math. 1034, Springer-Verlag, Berlin, Heidelberg, New York 1983.

[36] J. Musielak, "An application of modular spaces to approximation", Comment. Math., Tomus Specialis in Honorem Ladislai Orlicz, I(1979), 251259.

[37] J. Musielak, W. Orlicz, "On modular spaces", Studia Math. 18(1959), 49-65. 
[38] H. Nakano, " modulared semi-ordered spaces", Tokyo 1950.

[39] Z. Opial, "Weak convergence of the sequence of successive approximations for nonexpansive mappings", Bull. Amer. Math. Soc. 73(1967), 591-597.

[40] Z. Opial, "Nonexpansive and Monotone mappings in Banach spaces", Lecture Notes 67-1, Center for Dynamical Systems, Brown University, Providence, R.I., 1967.

[41] S. Prus, "Banach spaces with the uniform Opial property", Nonlinear Analysis, To appear.

[42] A.C. Zaanen, "Linear Analysis", North Holland/Amsterdam/Groningen, 1960. 